\title{
Male mating biology
}

\section{Paul I Howell $* 1,2$ and Bart GJ Knols²}

\begin{abstract}
Address: ${ }^{1}$ Centers for Disease Control and Prevention (CDC), 4770 Buford Hwy, Atlanta GA USA 30341, Malaria Research and Reference Reagent Resource Center (MR4) and Atlanta Research \& Education Foundation (AREF), 1670 Clairmont Road (151F), Decatur, GA 30033, USA and 2Div. Infectious Diseases, Tropical Medicine \& AIDS, Academic Medical Center, F4-217, Meibergdreef 9, 1105 AZ Amsterdam, The Netherlands and K\&S Consulting, Kalkestraat 20, 6669 CP Dodewaard, The Netherlands
\end{abstract}

Email: Paul I Howell* - bsr7@cdc.gov; Bart GJ Knols - bart@malaria-world.com

* Corresponding author

Published: 16 November 2009

Malaria Journal 2009, 8(Suppl 2):S8 doi:10.1186/1475-2875-8-S2-S8

This article is available from: http://www.malariajournal.com/content/8/S2/S8

(C) 2009 Howell and Knols; licensee BioMed Central Ltd.

This is an open access article distributed under the terms of the Creative Commons Attribution License (http://creativecommons.org/licenses/by/2.0),

which permits unrestricted use, distribution, and reproduction in any medium, provided the original work is properly cited.

\begin{abstract}
Before sterile mass-reared mosquitoes are released in an attempt to control local populations, many facets of male mating biology need to be elucidated. Large knowledge gaps exist in how both sexes meet in space and time, the correlation of male size and mating success and in which arenas matings are successful. Previous failures in mosquito sterile insect technique (SIT) projects have been linked to poor knowledge of local mating behaviours or the selection of deleterious phenotypes during colonisation and long-term mass rearing. Careful selection of mating characteristics must be combined with intensive field trials to ensure phenotypic characters are not antagonistic to longevity, dispersal, or mating behaviours in released males. Success has been achieved, even when colonised vectors were less competitive, due in part to extensive field trials to ensure mating compatibility and effective dispersal. The study of male mating biology in other dipterans has improved the success of operational SIT programmes. Contributing factors include inter-sexual selection, pheromone based attraction, the ability to detect alterations in local mating behaviours, and the effects of long-term colonisation on mating competitiveness. Although great strides have been made in other SIT programmes, this knowledge may not be germane to anophelines, and this has led to a recent increase in research in this area.
\end{abstract}

\section{Background}

Currently, control of malaria vectors focuses on the use of insecticide-treated bed nets and indoor residual spraying. Due to the rapid emergence and spread of insecticide resistance, alternative methods are needed to control vector populations. Many of these technologies, such as entomopathogenic fungi and viral paratransgenesis, have already shown great promise in laboratory settings but still have to be evaluated under field conditions [1-3].

An additional vector control method currently being reconsidered is the sterile insect technique (SIT). SIT is based on the concept of releasing sterilised, but sexually competitive males who will mate with wild females who thus lay sterile eggs which reduces vector populations and hence reduces or halts disease transmission [4]. Since the overall competitiveness of released vectors is expected to be below that of the wild insects and to accomplish an effect rapidly, an excess of sterile males relative to the number in the field have to be released. Although there have been many successes with other dipterans, earlier mosquito SIT programmes often produced conflicting results ([5] and [6]). Many failures have been linked to a lack of understanding of male mating biology, especially in regard to the ability of the mass-reared males to seek and mate with wild females [7]. Failures to control Culex 
tarsalis, Culex tritaeniorhynchus and Anopheles culicifacies were all correlated with inadequate knowledge of male mating behaviour [8].

Understanding mating in the context of the local vector population is likely to be paramount for success. Inferences about mating behaviours are limited as most of the information known is derived from studies using a limited number of tropical and temperate species. Very little is known about how and where males and females meet and what effect colonisation has on mating behaviours. The fundamental lack of knowledge in mating biology highlights the need to conduct research into how anophelines locate, identify, compete for and secure matings with conspecifics.

\section{Male sexual maturation}

There is a paucity of information available on the sexual behaviour of male anopheline mosquitoes. Most information is 30 years old and derived from research done mainly with colonised An. culicifacies, Anopheles gambiae, or Anopheles stephensi. From these early studies an incomplete picture of male sexual development and mating biology can be pieced together. Males are not competent to mate at emergence since terminalia, sexual organs and antennal fibrillae must first mature. During the first 12-24 $\mathrm{h}$ after emergence the male's terminalia undergo an inversion of $180^{\circ}$ in order to be properly oriented for mating [9]. Even though terminalia inversion is typically completed in $24 \mathrm{~h}$, males are still not sexually mature as reflected by a delay in sexual activity. Spermatogenesis is reported to begin in the late pupal stage $[10,11]$, however mating is not dependent on the presence of mature spermatozoa alone. Peak spermatogenesis in An. stephensi occurred between 0-5 days post emergence [12], however males became sexually active $48 \mathrm{~h}$ post emergence with peak mating activity 3-7 days post emergence [13]. Although An. gambiae and Anopheles arabiensis males were sexually active $48 \mathrm{~h}$ post-emergence, males younger than three days old had low rates of insemination [14] peaking seven days post emergence $[15,16]$. Mating in laboratoryreared An. gambiae was associated with the accumulation of male accessory gland (MAG) proteins even though sperm were present in the vesicles at $28 \mathrm{~h}$ post emergence [15]. Accumulation of MAG proteins requires 72-100 h $[15,17]$ and was noted to be coincident with increased mating activity in male anophelines $[12,13,15]$.

Additionally, full function of the male antennal fibrillae which is essential for mating does not occur until $12 \mathrm{~h}$ post emergence. Erect fibrillae respond to female flight tones and are required for location of females $[9,18]$. Three to four day old male An. stephensi were most responsive to female wing beat sounds [19]. Sexually mature male An. gambiae mosquitoes that had one or both their antennae removed were unable to locate females to mate $[14,19]$. Receptivity to wing beat tones, therefore, appears to be concordant with sexual maturation in males.

\section{Mate location}

Mating in most anopheline mosquitoes is often believed to take place only in swarms $[20,21]$ although a recent report suggests indoor mating in African anophelines [22]. Marchand [23] hypothesized that positive phototaxis in An. gambiae males occurs typically between 300500 lux and primarily at dusk. Similar ranges have been observed in other anophelines. Light changes from 592 lux to 18.3 lux stimulated An. culicifacies males to mate in the laboratory [24] while in nature it was reported to be between 467.2 to 26.9 lux [25]. Anopheles freeborni males commenced swarming around 350 lux and dispersed around 0.5 lux [26]. The onset of mating is not related simply to the decrease in light intensity but is also tied to the inherent circadian rhythm in anophelines. Although lux values associated with mating occurred during the day, Anopheles franciscanus did not swarm until sunset [27]. Charlwood [14] showed a reduction in inseminations by males that had their circadian rhythm altered.

The interplay of circadian rhythm, swarming stimulation, and light level is reflected by the disparate lux values associated with the onset of mating. These interspecific differences may serve as mating barriers. In Pakistan, wild anophelines were found to commence swarming over the same markers but at different times [28]. However, in the laboratory, four members of the An. gambiae complex were found to have differing active periods with some overlap between sibling species [29]. Therefore, although swarming may be initiated at different times in some species, other mechanisms, such as spatial factors, must act to isolate species with overlapping swarming periods.

Swarms have been reported to form at varying heights as well, possibly determined by species-appropriate swarm markers. Markers are visually evident objects or contrasting areas either on the ground or horizon used for orientation during swarming [20]. There is some difficulty in associating specific markers with swarming males, or even being certain that they exist, as many natural ones are not evident to the observer. An. gambiae has been observed swarming between 2-3 m over no discernable marker [30] and 1-4 m [31] above ground markers. An. gambiae s.l. swarms were observed between $0.5-2 \mathrm{~m}$ with no discernable markers [23]. Anopheles funestus males formed swarms at heights of 2-4 m employing horizon markers [32]. Sympatric populations of Anopheles hyrcanus and Anopheles philippenensis swarmed at a height of 0.3-0.6 m above small shrubs and flowers (ground markers) and 1.5-2.5 m in large open spaces (horizon markers) [33]. 
Swarming height may also be related to orientation with swarm markers. Male S-form An. gambiae swarm near female feeding sites with an unobstructed view of the sky [23]. An. funestus swarms appeared to rely more on horizon markers [32] whereas An. gambiae and Anopheles merus relied more on ground and horizon markers [22,31,34]. Similarly, An. freeborni utilized both horizon and ground markers [26], and An. culicifacies males preferred to swarm over low mounds. Indoor-mating $\mathrm{M}$-form An. gambiae oriented themselves in doorways and near eaves irrespective of any ground markings [22]. Swarming at different heights, therefore, may act as another barrier to prevent inter-species mating. Several genera of insects have been reported to swarm during the same period over the same marker [31], even then therefore, aggregations formed at varying heights could result in inter-species segregation.

Although much work has been done to elucidate what constitutes a swarm site, it has yet to be determined why males of different species choose certain areas. The need for a marker has been shown in several studies though none is evident in others. When a potential horizon marker was obscured, swarms of An. funestus were seen to gain altitude in order to re-orient themselves in the same place [32]. Similarly, swarms followed large cloth ground markers that were moved for short distances [34]. Potential swarm markers that were removed, resulted in the abandonment of that site by males [31]. In contrast, placing visual markers under swarms of An. funestus apparently led to displacement of the swarm (J.D. Charlwood, pers. comm. [32]).

Therefore, mosquitoes locate themselves in space and time to ensure they are available to mate. The interplay of time of initiation, marker type, and height in swarm formation reduce the probability of intraspecific mating [20]. Mixed species swarms are rare even in areas where sympatric species occur. In the field a limited number of heterospecific individuals have been collected during swarm sampling. The $\mathrm{M}$ - and S-forms of An. gambiae swarm at similar times, however they are rarely ever encountered in the same area [30,35]. Similarly, of $31 \mathrm{An}$. gambiae swarms sampled in Tanzania only five contained other anophelines [23]. Cunningham-van Someren routinely collected large swarms of Anopheles squamosus in Kenya that contained no more than one or two individuals of other anophelines [36]. The difference in marker recognition, resulting in intra-specific swarming, was shown between An. gambiae and An. funestus in Mozambique [32]. However, what constitutes an appropriate mating arena or what features are used for orientation for many species remains elusive.

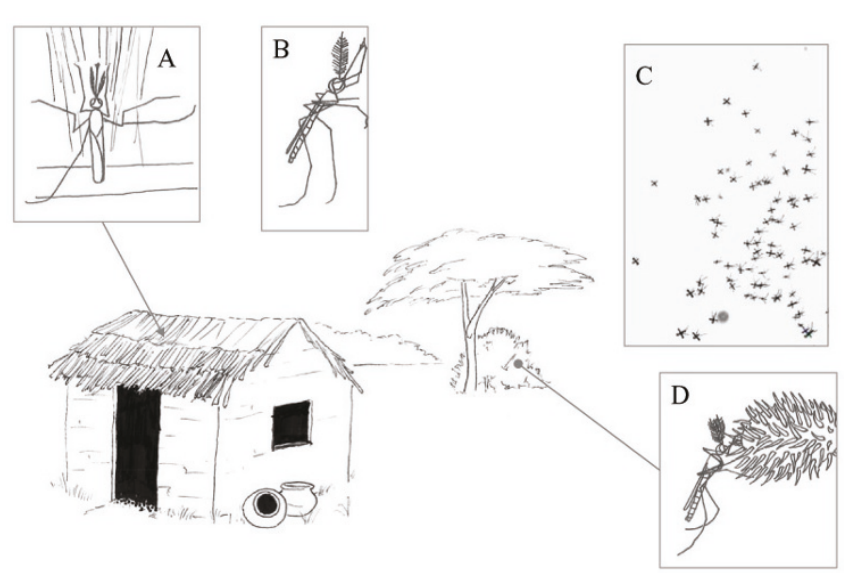

\section{Figure I}

The sexually mature male: $A$. diurnal indoor resting, B. I hour prior to swarming the antennal fibrillae become erect, C. males depart to commence swarming, D. After swarming ceases, male ingest a sugar meal prior to resuming indoor resting.

\section{Swarming and mating behaviour of anophelines} Sexually mature male mosquitoes leave their diurnal resting sites at species specific times to commence swarming (Figure 1). The antennal fibrillae become erect and typically remain so for 1-2 h [14], however in laboratoryreared An. stephensi this period lasted up to six hours [37]. Swarming commenced at various times for different species $[25,26,30,34]$. As dusk nears, males begin to fly over the swarming arena [25,34]. Gradually more males join forming large, loose aggregations. Eventually the males move from a distended, non-specific circling motion into a more rigid, condensed group $[25,34]$. Swarms are comprised mainly of males (Table 1 ) with numbers varying from approximately 5-5000 individuals.

Both in the laboratory and in the field, females become active after the males. Hence copulations are not usually observed until 5-20 min after swarm initiation. In Malaysia, An. philippinensis started mating 5 min after swarming commenced while An. hyrcanus began mating 10 min later [33]. An. gambiae and An. funestus males started mating approximately $10 \mathrm{~min}$ after swarming began [26] and An. freeborni commenced mating 5-10 min after swarming began. It is unknown why males appear at swarming arenas before females. Females may arrive later than males due to different phototactic stimuli. This may also be a behavioural response by males to ensure that they arrive at mating arenas before females to optimize their chances for mating.

Once formed, the swarm begins to move as a single unit (Figure 2). Male An. gambiae were observed to be unevenly 
Table I: Captured anopheline males and females from various wild swarms

\begin{tabular}{|c|c|c|c|c|c|}
\hline Species & males & females & max. \% of females & Avg. number swarming & Reference \\
\hline An. culicifacies & 715 & 5 & 7.0 & nd & [24] \\
\hline An. culicifacies & 544 & 14 & 2.4 & $15-3000$ & {$[25]$} \\
\hline An. franciscanus & 2341 & 3 & 0.1 & $1000-5000$ & {$[27]$} \\
\hline An. freeborni & 6028 & 175 & 8.0 & $300-1500$ & [26] \\
\hline An. gambiae (M) & 2823 & 6 & 0.1 & nd & [30] \\
\hline An. gambiae (S) & 511 & 6 & 4.0 & nd & [35] \\
\hline An. squamosus & 611 & 0 & 0.0 & $40-300$ & [36] \\
\hline An. stephensi & 1061 & 18 & 2.8 & nd & [59] \\
\hline An. subpictus & 2242 & 157 & 20.0 & $75-500$ & [98] \\
\hline
\end{tabular}

nd: No data given by author

distributed within the swarm with more males occupying the centre versus the periphery [38]. This centralized positioning may increase a males' chance to acquire a mate by either occupying the space most frequented by females or being more likely to better detect approaching females. It may also be a function of males aligning themselves with a potential swarm marker.

Swarming males have been reported to either mate with females as they penetrate the group $[31,32,34]$ or as they temporarily departed to secure a female flying nearby $[23,25,39]$. Approaching females may be detected by their wing beat frequencies, which is typically lower than that of males. The range for detection is unknown, but in the laboratory An. gambiae males could detect a female at distances of 50-75 mm [14]. The ability of the male to detect

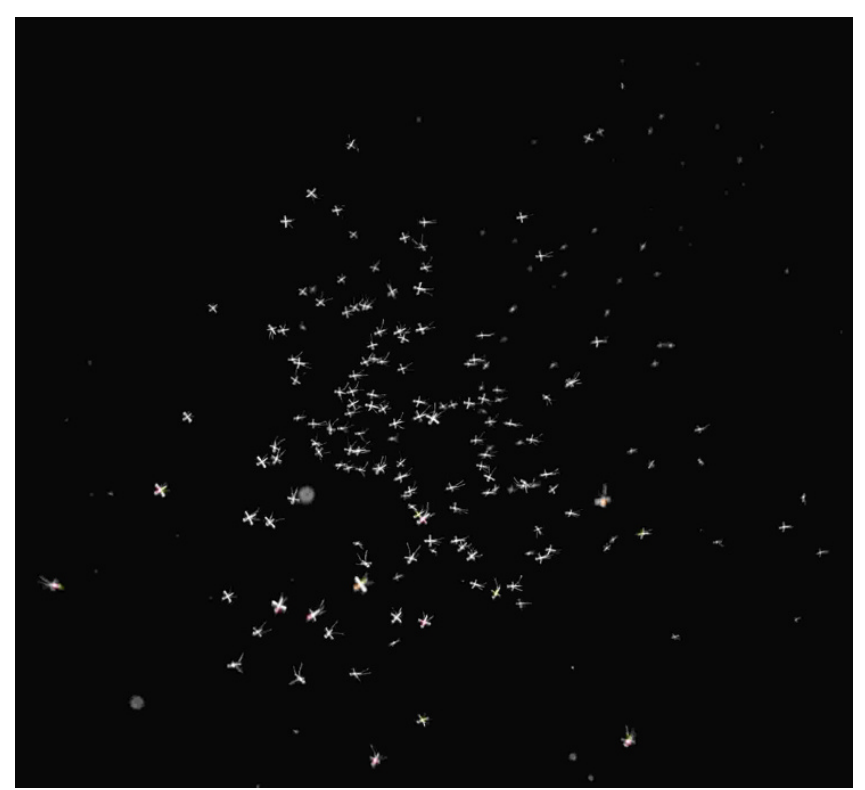

Figure 2

Anopheles pharoensis Theobald swarming (Photo courtesy of J.D. Charlwood). conspecific female wing beats and reject heterospecific females has been proposed as another barrier to interspecific mating in An. gambiae s.l. [40]. Males of some mosquito species attempt to harmonize their wing beats with nearby insects (sexual tuning) to determine if they are conspecific and of the opposite sex [41] although this remains unknown for anophelines. Wild populations, however, display a greater variance in their tones resulting in widespread overlap and reduced discrimination of wing beat sound as a single way for males to identify conspecific females at a distance $[42,43]$.

The actual period during which mating occurs in the swarm is brief. Once mating commences, copulating pairs are seen usually for 10-30 min as darkness approaches $[30,32,35]$. This shortened mating period may be related to the length of time spent in copula. Copulation in An. gambiae lasts 15-20 s [14] and approximately $27 \mathrm{~s}$ in An. culicifacies [25].

Detailed studies on copulation in Anopheles have shown that as a male approaches the female he grasps her with his tarsal claw on his first pair of legs and then swings his abdomen up to clasp her genitalia. Once interlocked, the male releases his tarsal grasp of the female and they assume the venter-to-venter position and resume flying $[14,20,44]$. The copulating pair then departs the swarm and flies off to complete the mating after which the male is assumed to rejoin the swarm. It is unknown if males will mate again on the same night, however observations exist of anopheline males returning to a swarm after mating (J.D. Charlwood, pers. comm. [26]).

Male courtship is considered to be absent in many swarming insects [45]. Instead, when females are a limiting factor and the costs of mate finding are high, males may forgo "choosy" behaviours so as to not waste potential mating opportunities [46]. This results in a "no-choice" scenario for both sexes as males cannot refuse a mate based on some phenotypic character and females will have limited choice, if any, in accepting a male's advances. 
There are no reported field observations of swarming males seeking out and rejecting a female. Possible indiscriminate mate choice by males was reported in wild $A n$. funestus. Wing lengths of females caught in copula were not different from those of newly emerged females [32]. Therefore, males are likely to mate with females of unknown quality as their chances of meeting and copulating are low. In contrast, tethered virgin females have been reported to dislodge males attempting to copulate in the laboratory [14].

It remains unknown if males are capable of assessing female phenotypic quality and differentially allocate copulatory resources based on female quality during mating [46]. Due to low female availability, the reported high risk of predation during swarming, and the energy budget required for doing so, males will have limited opportunities to mate [21]. Therefore, it would benefit males to invest in quality inseminations thereby imposing monogamy on females via the insertion of a large volume of sperm and a mating plug. Natural behaviour corroborates this as polyandry is uncommon in wild An. gambiae $(2.5 \%)$ (reviewed in $[21,47])$. However, males have been reported to resume swarming after mating. Therefore it may be possible for males to achieve a second mating on the same evening. This would mean that males might not be investing in one large ejaculate that depletes their capacity, but several smaller ones to ensure that they may mate with quality females. Indeed, laboratory reared $A n$. gambiae are capable of inseminating up to five females, of which two included sperm and mating plugs [48].

Mating without swarming has been reported in several anophelines. Mating in alternate venues is often employed by individuals who are competitively disadvantaged [22]. As in other insect groups, smaller males or males who are physiologically stressed and not capable of swarming for long periods of time may adopt alternate mating strategies to ensure they pass on their genes [22]. Since the male antennal fibrillae become erect one hour before swarming commences, males should be sexually responsive to females in the vicinity even when not swarming ([23] and reported in [34]). Alternate venues may also favour sexual choice by allowing individuals a chance to select mates based on phenotypic qualities. Since females are considered to be monogamous, pre-copulatory choice would prevent mating with lesser quality males.

Once mating is completed, the female is assumed to become refractory to further mating due to the transfer of several male compounds. After ejaculation, males insert a mating plug composed of MAG substances [49]. In other culicids, MAG substances are responsible for causing the switch of female behaviour from unmated to mated. This was originally reinforced by reported polyandry in $A n$. gambiae s.l. due to the inability of hybrid males with abnormal MAGs to prevent remating [50]. However, in the laboratory it was determined that the presence of sperm in the spermatheca is responsible for the switchover state in anophelines [51,52]. Though the function of MAG proteins in anophelines is unknown, several malespecific accessory gland proteins that are known to trigger post mating behavioural changes in Drosophila [49] have recently been identified in An. gambiae. Similarly, the insertion of the mating plug, and its associated substances, has been shown to turn off the mating machinery in An. gambiae females possibly resulting in a physiological barrier to remating [53]. Although superficially contradictory to earlier work $[49,50]$, the processing of the MAG substances within the female's atrium may be necessary to stimulate post-mating processes, which might have been inadvertently by-passed in the original experiments [51].

The number of matings occurring in the swarm declines until darkness when most males disperse to rest and replenish energy reserves through nectar feeding. Males were found to expend up to $50 \%$ of their energy reserves during swarming and to feed shortly before dawn [54]. This meal was used mainly to fuel flight during the period prior to scotophase [55]. Nocturnal feeding, therefore, is required by males to replenish energy reserves utilized during mate seeking as well as for daily survival.

It has been argued that swarming is ritualistic and not important in mating (reviewed in [20]). Swarming without mating $[23,28]$ or infrequent pairing $[23,28,32,34]$ has been observed for many species. However, a great number of copulating pairs have been observed in swarms of these same species $[31,39]$. The crepuscular nature of swarming often hinders the researcher's ability to observe actual mating. Similarly, the number of sexually mature males far outnumbers the number of virgin females on any given evening [34]. This would result in an underestimation of the actual number of matings. Swarming may still be the most efficient way for males and females to meet in highly dispersed populations.

It is, therefore, unknown what male or female phenotypic characters, if any, are associated with mating success. Similarly, of the males that do mate, it remains unknown whether they copulate with several females to increase their chance of securing a high quality female (i.e. cryptic male choice).

\section{Hindrances to male mating}

After sexual maturation, opportunities for a male to mate are limited by several factors. First, sugar feeding is important to both male survival and mating ability. Due to their small energy reserves relative to females, teneral males 
must locate a carbohydrate source in order to survive to sexual maturity [56]. Mortality in unfed An. gambiae males was reported to start at 24-29 h post emergence with $82 \%$ deceased within $48 \mathrm{~h}$ [57]. Concordant with field observations, males fed throughout the night in the laboratory and rested without imbibing during the day [58]. Not every male will feed every night; therefore the ability of a male to successfully locate a nectar source will be linked to its ability to swarm and mate [55].

Mating opportunities for older males may not occur either due to predation or diminished mating abilities. Colonised anophelines lived up to 20 days but based on recapture experiments it was estimated that the average lifespan of wild males was between 5-10 days $[59,60]$ of which the first 1-2 days are devoted to sexual maturation. Although older males could represent a more genetically fit population [61], females mated to six day old males were less likely to oviposit than those mated to two day old males in the laboratory [15]. However, Reisen [62] found that there were similar numbers of mated and unmated males in both the 0-5 days and 5 day plus age groups. Reluctance for older males to mate, even though they still have spermatozoa in their testes, may be behavioural in nature and not a result of senescence $[15,24]$. In conclusion, it is unknown if older males are more or less likely to mate than their younger counterparts.

Next, not every male will have an opportunity to mate during its lifetime. In An. stephensi only $42 \%$ of males copulated in the laboratory [13]. Based on male sex organ dissections, ejaculation rates in wild male An. culicifacies varied from $14-47 \%$ with unmated individuals found in all age groups [62]. Smaller male An. freeborni had less success than their larger counterparts in inseminating females in swarms [26]. Certain males may be more fit than others - be it size or age - allowing them to secure more matings during their lifetime while less fit individuals acquire none. Also, the ability of certain males to replenish spermatozoa and MAGs after mating may limit the number of copulations achieved [13,62]. Early swarming males are also prone to higher predation rates [32,63]. Finally, the number of sexually mature, virgin females will be less than the number of sexually mature males available nightly $[23,35]$. Therefore, disproportionate mating opportunities and abilities combined with a lack of sexually receptive females would result in failure for some males to successfully mate.

The role of male size has been studied with interesting but inconsistent results with regard to mating success in anophelines. In An. freeborni, larger males mated more successfully than their smaller counterparts [26]. Conversely, in An. funestus males that were collected during swarming and in copula were no larger than those col- lected from indoor resting sites [32]. In An. gambiae there is conflicting evidence regarding male and female size and its influence on mating success [64-67]. One would not expect male size to be an important factor in swarm-based mating. In a no-choice situation males cannot risk refusing a female in hopes of finding a better quality mate [46].

Anopheline male mating success, therefore, relies on several parameters whose values are scarcely known and only for a few species. Male size does have an effect on reproductive abilities in temperate anophelines. Its effects, however, are unknown in tropical species, which represents an area that is in need of further research. What is known from laboratory studies and wild observations is that sexual maturation requires 1-2 days and males older than seven days are typically reluctant to mate. Therefore, a normal anopheline male may accomplish between 0-3 matings in his lifetime.

\section{The effects of colonisation on the mating behaviour of males}

Released males must be able to 1) disperse over considerable ranges, 2) locate appropriate mating venues, and 3) compete with feral males [4]. Quality control protocols in the insectary are used to ensure that males achieve a reasonable level of quality, at least in relation to efficient mass rearing $[68,69]$. However these same protocols often act antagonistically in regards to dispersal and mating of the males in the field. Colonisation of vectors often leads to the inadvertent or purposeful fixation of alleles, which affect mating behaviours. Even when efforts are made to retain either large numbers of vectors, introduce wild material to maintain polymorphism, or by rearing under semi-natural conditions, colonisation will lead to behavioural changes in the vector [70-72]. The unnatural environment of the insectary led to a quick fixation of alleles or behaviours that resulted in assortative mating between colonised and native populations which were evident within four generations [73].

The establishment of a laboratory colony typically results in a bottle-neck due to the selective pressures placed on individuals to adapt to the unnatural environment. Unless an out-breeding scheme is implemented, this is even more pronounced during the establishment of a genetic sexing strain or a specific transgenic strain in which only one founder individual will have the correct phenotype. Kaneshiro [74] hypothesized that these bottle-necked subpopulations have altered their mate selection standards in an effort to avoid extinction. Normal mating behaviours may not be possible within a cage due to space limitations. This results in shortened or modified mating behaviours, which may be less desirable in the wild. Colonised insects therefore may become less "choosy" in mate selection: colonised insects would cop- 
ulate with both laboratory-derived and wild insects while wild insects would reject matings with laboratory insects due to their unnatural mating behaviours.

Standardized rearing protocols are necessary to produce insects of known quality, and the environmental parameters employed typically do not recreate field conditions. This may lead to confusion of the male in regards to when or where to mate. In Cochliomyia hominivorax, it was reported that laboratory released flies were active later in the day than wild flies which were more active in the morning resulting in decreased encounters between the two populations [75]. Conversely, released Bactrocera cucurbitae were found to be more active earlier in the day while wild flies were active later [76]. In An. gambiae it was found that light bursts or alterations to the LD period caused disruptions in male flight activity [29,77]. An. culicifacies reared under standardized conditions failed to swarm properly in the wild for two days post-release [78], but swarming and mating were synchronized with wild mosquitoes when this strain was reared under a more natural photoperiod. In the laboratory, mating success of wild mosquitoes required both proper light intensity and the presence of a swarm marker while laboratory colonies mated indiscriminately [79].

Additionally, geographically distinct populations may exhibit different mating abilities. The accumulation of genetic differences necessary for survival and reproduction in different niches leads to ecological divergence and can result in pre-zygotic isolation [80]. Although geographic mating polymorphism has been reported between three colonies of An. stephensi $[13,81]$, it has not been found to be a limiting factor in mating in An. arabiensis[82]. A population of An. arabiensis from La Réunion Island mated freely with continental African colonies. Therefore, efforts to discern the effects of geographic barriers to mating between disparate populations, as done in the Mediterranean fruit fly Ceratitis capitata SIT programme, would be necessary to ensure any colony established does not suffer from assortative mating [83].

Finally, colonisation has been associated with assortative mating behaviours. Colonised $C x$. tarsalis released into an isolated area swarmed in different arenas compared to wild vectors [84]. Reisen [8] noted that even when a test population had a similar genetic background, assortative mating was still seen when released into the wild. It was concluded that assortative mating was the consequence of colonisation and that it occurred within 3-4 generations [73]. Even when released with laboratory reared, wild caught progeny, colonised vectors were discriminated against [85]. Chemosterilised An. culicifacies males were non-competitive in nature even though they rested and swarmed concomitantly with wild males and were equally competitive in laboratory trials [62]. Previous reports of assortative mating in released anophelines have involved long-colonised strains $[62,86,87]$. However, as reported by Kaiser et al. [88] and Helinski et al. [89], wild females did not discriminate against released males from recently colonised lines.

\section{Conclusion}

In the last decade, the role of male mating biology has been repeatedly mentioned as a major limitation to any mass release programmes of sterile insects [7,8,90,91]. The success in controlling other dipterans utilizing SIT has largely been due to the extensive study of male mating biology [92-94]. Therefore, a fundamental knowledge of how males aggregate, encounter females, and copulate as well as techniques to detect alterations in mating behaviours is necessary.

Extensive field-testing is necessary to determine the level of mating competence between colonised vectors and native populations. Entraining colonised vectors to the local environment may increase success. Exposing teneral males to chemical or environmental cues may help condition them to locate sites where females are known to feed or mate. Males may locate mating arenas through experiences gained during their sexually immature period: site fidelity and memory have been suggested in dipterans such as An. arabiensis [95]. Teneral colony males could be housed near potential release sites. This would expose males to local parameters such as photoperiod, nectar sources, and environmental conditions to ensure they are capable of survival after release. The filter rearing system employed in the C. capitata SIT programme exposes colonised insects to more natural environmental conditions, natural host plants, and more natural rearing parameters resulting in a higher quality mating strain $[68,96]$.

Lastly, is it possible to maintain a genetically modified colony long-term without detriment to mating behaviours? Colonisation of insect vectors for control programmes often leads to a "paradox of genetic breeding": the higher the output typically the lower the quality [97]. Although success in both medfly and melon fly SIT programmes have been attributed to long term colonisation without diminished mating abilities [93,96], planning should be undertaken to optimize rearing conditions with minimal alteration to "normal" abilities or behaviours. Optimization of rearing conditions, however, should be done with great caution so inadvertent selection of nonbeneficial phenotypes is avoided.

\section{Key research questions}

Before implementing an SIT programme, there are several points that need further research in order to increase the likelihood of success, especially in regard to An. arabiensis. 
- Which phenotypic characters are associated with male mating success?

- Do males allocate all of their resources into one mating or instead invest in several smaller ejaculates?

- Do males swarm every night of their lives?

- Which mating behaviours are altered during colonisation and can these be avoided or their effects lessened?

- Will entraining vectors to local conditions prior to release increase their mating success?

- Will the geographic origin of the mosquito lead to assortative mating?

- How can we optimize rearing conditions to make a more competitive mosquito?

\section{Competing interests}

The authors declare that they have no competing interests.

\section{Authors' contributions}

PIH wrote the manuscript. BGJK critiqued and revised all sections. Both authors read and approved the final manuscript.

\section{Acknowledgements}

Thank you to Heather Ferguson, Mark Benedict, Alan Robinson and Boaz Yuval for their constructive comments on this paper and J.D. Charlwood for his personal insight into this topic and his photograph of swarming mosquitoes.

This article has been published as part of Malaria Journal Volume 8 Supplement 2, 2009: Development of the sterile insect technique for African malaria vectors. The full contents of the supplement are available online at http://www.malariajournal.com/supplements/8/S2.

\section{References}

I. Catteruccia F: Malaria vector control in the third millennium: progress and perspectives of molecular approaches. Pest Manag Sci 2007, 63:634-640.

2. Scholte EJ, Knols BGJ, Samson RA, Takken W: Entomopathogenic fungi for mosquito control: a review. J Insect Sci 2004, 4: I9.

3. Takken W, Knols BGJ: Malaria vector control: Current and future strategies. Trends Parasitol 2009, 25:101-104.

4. Knipling EF, Laven H, Craig GB, Pal R, Smith CN, Brown AWA: Genetic control of insects of public health importance. Bull World Health Organ 1968, 38:42I-438.

5. Benedict $M Q$, Robinson $A S$ : The first releases of transgenic mosquitoes: an argument for the sterile insect technique. Trends Parasitol 2003, 19:349-355.

6. Dame DA, Curtis CF, Benedict MQ, Robinson AS, Knols BG]: Historical applications of induced sterilization in field populations of mosquitoes. Malar J 2009, 8(Suppl 2):S2.

7. Ferguson HM, John B, Ng'habi K, Knols BGJ: Redressing the sex imbalance in knowledge of vector biology. Trends Ecol Evol 2005, 20:202-209.

8. Reisen WK: Lessons from the past: historical studies by the University of Maryland and the University of California, Ber- keley. In Ecological Aspects for Application of Genetically Modified Mosquitoes Edited by: Takken W, Scott TW. Dordrecht: Kluwer; 2003:25-32.

9. Clements AN: The Biology of mosquitoes. Sensory Reception and Behavior Volume 2. London: $\mathrm{CABI}$; 1999.

10. Barr AR: Symposium on reproduction of arthropods of medical and veterinary importance. V. Reproduction in Diptera of medical importance with special reference to mosquitoes. J Med Entomol 1 974, I I:35-40.

II. Helinski ME, Parker AG, Knols BGJ: Radiation biology of mosquitoes. Malar J 2009, 8(Suppl 2):S6.

12. Mahmood F, Reisen WK: Anopheles culicifacies: effects of age on the male reproductive system and mating ability of virgin adult mosquitoes. Med Vet Entomol 1994, 8:31-37.

13. Mahmood F, Reisen WK: Anopheles stephensi (Diptera: Culicidae): changes in male mating competence and reproductive system morphology associated with aging and mating. J Med Entomol 1982, 19:573-588.

14. Charlwood JD, Jones MDR: Mating behaviour in the mosquito, Anopheles gambiae s.l. I. Close range and contact behaviour. Physiol Entomol 1979, 4: I II-I20.

15. Chambers GM, Klowden MJ: Age of Anopheles gambiae Giles male mosquitoes at the time of mating influences female oviposition. J Vector Ecol 200I, 26: 196-201.

16. Verhoek BA, Takken W: Age effects on the insemination rate of Anopheles gambiae s.I. in the laboratory. Entomol Exp Appl 1994, 72:167-172.

17. Mahmood F: Age-related changes in development of the accessory glands of male Anopheles albimanus. J Am Mosq Control Assoc 1997, 13:35-39.

18. Nijhout HF, Sheffield HG: Antennal hair erection in male mosquitoes: a new mechanical effector in insects. Science 1979, 206:595-596.

19. Ikeshoji T: Age structure and mating status of the male mosquitoes responding to sound. Eisei Dobutsu Jap J Sanit Zool 1985, 36:95-10I.

20. Downes JA: The swarming and mating flight of Diptera. Annu Rev Entomol 1968, I 4:271-298.

21. Yuval B: Mating systems of blood-feeding flies. Annu Rev Entomol 2006, 5 1:413-440.

22. Dao A, Adamou A, Yaro AS, Alpha SY, Hamidou MM, Kassogue $Y$, Traore SF, Lehmann T: Assessment of alternative mating strategies in Anopheles gambiae: Does mating occur indoors? Med Entomol 2008, 45:643-652.

23. Marchand RP: Field observations on swarming and mating in Anopheles gambiae mosquitoes in Tanzania. Neth J Zool 1984, 34:367-387.

24. Reisen WK, Mahmood F, Parveen T: Laboratory observations on the time of mating of Anopheles culicifacis Giles. Mosq News 1979, 39:328-333.

25. Reisen WK, Aslamkhan M: Observations on the swarming and mating behaviour of Anopheles culicifacies Giles in nature. Bull World Health Organ 1976, 54: I55- 158.

26. Yuval B, Wekesa JW, Washino RK: Effect of body size on swarming behaviour and mating success of male Anopheles freeborni (Diptera: Culicidae). J Insect Behav 1993, 6:155-158.

27. Belkin JN, Ehmann N, Heid G: Preliminary field observations on the behavior of the adults of Anopheles franciscanus McCracken in southern California. Mosq News 1951, I I:23-31.

28. Reisen WK, Aslam Y, Siddiqui TF: Observations on the swarming and mating of some Pakistan mosquitoes in nature. Ann Entomol Soc Am 1977, 70:988-995.

29. Jones MDR, Gubbins SJ: Circadian flight activity in four sibling species of the Anopheles gambiae complex (Diptera, Culcidae). Bull Entomol Res 1974, 64:24I-246.

30. Diabate A, Baldet T, Brengues $C$, Kengne $P$, Dabire KR, Simard $F$, Chandre F, Hougard JM, Hemingway J, Ouedraogo JB, Fontenille D: Natural swarming behaviour of the molecular $M$ form of Anopheles gambiae. Trans R Soc Trop Med Hyg 2003, 97:713-716.

31. Charlwood JD, Pinto J, Sousa CA, Ferreira C, Do Rosario VE: Male size does not affect mating success (of Anopheles gambiae in Sao Tome). Med Vet Entomol 2002, 16:109-1II.

32. Charlwood JD, Thompson R, Madsen H: Observations on the swarming and mating behaviour of Anopheles funestus from southern Mozambique. Malar J 2003, 2:2. 
33. Wharton RH: The habits of adult mosquitoes in Malaya. IV. Swarming of anophelines in nature. Ann Trop Med Parasitol 1953, 47:285-290.

34. Charlwood JD, Jones MDR: Mating in the mosquito, Anopheles gambiae. s.I. II. Swarming behaviour. Physiol Entomol 1980, 5:315-320.

35. Diabate A, Dabire RK, Kengne P, Brengues C, Baldet T, Ouari A, Simard F, Lehmann T: Mixed swarms of the molecular $\mathbf{M}$ and $\mathbf{S}$ forms of Anopheles gambiae (Diptera: Culcidae) in sympatric area from Burkina Faso. J Med Entomol 2006, 43:480-483.

36. Cunningham-van Someren GR: Male mosquito swarms: some observations in Kenya. Proc R Entomol Soc Lond A 1965, 40:89-91.

37. Nijhout HF: Control of antennal hair erection in male mosquitoes. Biol Bull 1977, 153:59I-603.

38. Manoukis NC, Diabate A, Abdoulaye A, Diallo M, Dao A, Yaro AS, Ribeiro JMC, Lehmann T: Structure and dynamics of male swarms of Anopheles gambiae. J Med Entomol 2009 in press.

39. Quraishi MS: Swarming, mating, and density of Anopheles stephensi mysorensis. J Econ Entomol 1965, 58:82 I-824.

40. Brogdon WG: Measurement of flight tone differences among members of the Anopheles gambiae species complex (Diptera: Culicidae). J Med Entomol 1998, 35:68I-684.

4I. Gibson G, Russel I: Flying in tune: sexual recognition in mosquitoes. Curr Biol 2006, 16:1311-1316.

42. Tripet F, Dolo G, Traore S, Lanzaro GC: The "wingbeat hypothesis" of reproductive isolation between members of the Anopheles gambiae complex (Diptera: Culicidae) does not fly. I Med Entomol 2004, 41:375-384.

43. Wekesa JW, Brogdon WG, Hawley WA, Besansky NJ: Flight tone of field-collected populations of Anopheles gambiae and An. arabiensis (Diptera: Culicidae). Physiol Entomol 1998, 23:289-294.

44. Omar AH, Shaurub EH: Mating behaviour and insemination in Anopheles multicolor Cambouliu. Journal of the Egyptian Society of Parasitology 1987, 17:38I-387.

45. Shelly TE, Whittier TS: Lek behaviour of insects. In The Evolution of Mating Systems in Insects and Arachnids Edited by: Choe JC, Crespi BJ. Cambridge: Cambridge University Press; 1997:273-293.

46. Bonduriansky $R$ : The evolution of male mate choice in insects: a synthesis of ideas and evidence. Biol Rev 200I, 76:305-339.

47. Tripet F, Touré YT, Dolo G, Lanzaro GC: Frequency of multiple inseminations in field collected Anopheles gambiae females revealed by DNA analysis of transferred sperm. Am J Trop Med Hyg 2003, 68: I-5.

48. Giglioli ME: The female reproductive system of Anopheles gambiae melas. I. The structure and function of the genital ducts and associated organs. Riv Malariol 1963, 42:149-176.

49. Dottorini T, Nicolaides L, Ranson H, Rogers DW, Crisanti A, Catteruccia F: A genome-wide analysis in Anopheles gambiae mosquitoes reveals 46 male accessory gland genes, possible modulators of female behavior. Proc Natl Acad Sci USA 2007, 104:16215-16220.

50. Bryan JH: Further studies on consecutive matings in the Anopheles gambiae complex. Nature 1972, 239:519-520.

51. Klowden MJ: Sexual receptivity in Anopheles gambiae mosquitoes: absence of control by male accessory gland substances. J Insect Physiol 2001, 47:66I-666.

52. Klowden MJ: Switchcover to the mated state by spermathecal activation in female Anopheles gambiae mosquitoes. I Insect Phys 2006, 52:679-684.

53. Rogers WD, Whitten MMA, Thailayil J, Soichot J, Levashina E, Catteruccia F: Molecular and cellular components of the mating machinery in Anopheles gambiae females. Proc Natl Acad Sci USA 2008, 105:19389-19394.

54. Yuval B, Holliday-Hanson ML, Washino RK: Energy budget of swarming male mosquitoes. Ecol Entomol 1994, 19:74-78.

55. Yuval B: The other habit: sugar feeding by mosquitoes. Bull Soc Vector Ecol 1992, 17:105-156.

56. Briegel H: Fecundity, metabolism, and body size in Anopheles (Diptera: Culicidae), vectors of malaria. J Med Entomol 1990 27:839-850.

57. Foster WA, Takken W: Nectar-related vs. human-related volatiles: behavioural response and choice by female and male Anopheles gambiae (Diptera: Culicidae) between emergence and first feeding. Bull Entomol Res 2004, 94: 145-157.

58. Gary RE Jr, Foster WA: Diel timing and frequency of sugar feeding in the mosquito Anopheles gambiae, depending on sex, gonotrophic state and resource availability. Med Vet Entomol 2006, 20:308-316.

59. Reisen WK, Aslamkhan M: A release-recapture experiment with the malaria vector, Anopheles stephensi Liston, with observations on dispersal, survivorship, population size, gonotrophic rhythm and mating behaviour. Ann Trop Med Parasitol 1979, 73:25I-269.

60. Reisen WK, Sakai RK, Baker RH, Azra K, Niaz S: Anopheles culicifacies: observations on population ecology and reproductive behavior. Mosq News 1982, 42:93-101.

6I. Huho BJ, Ng'habi KR, Killeen GF, Nkwengulila G, Knols BGJ, Ferguson $H M$ : Nature beats nurture: a case study of the physiological fitness of free-living and laboratory-reared male Anopheles gambiae s.l. J Exp Biol 2007, 21 0:2939-2947.

62. Reisen WK, Baker RH, Sakai RK, Mahmood F, Rathor HR, Raana K Toqir G: Anopheles culicifacies Giles: mating behavior and competitiveness in nature of chemosterilized males carrying a genetic sexing system. Ann Entomol Soc Am I98I, 74:395-40I.

63. Yuval B, Bouskila A: Temporal dynamics of mating and predation in mosquito swarms. Oecologia 1993, 95:65-69.

64. Gomulski LM: Larval density, adult size and mating competitiveness in the mosquito Anopheles gambiae. Trans $R$ Soc Trop Med Hyg 1985, 79:276-277.

65. Ng'habi KR, John B, Nkwengulila G, Knols BGJ, Killeen GF, Ferguson HM: Effect of larval crowding on mating competitiveness of Anopheles gambiae mosquitoes. Malar 」 2005, 4:49.

66. Ng'habi KR, Huho BJ, Nkwengulila G, Killeen GF, Knols BGJ, Ferguson $\mathrm{HM}$ : Sexual selection in mosquito swarms: may the best man lose? Anim Behav 2008, 76: 105-112.

67. Okanda FM, Dao A, Njiru BN, Arija J, Akelo HA, Touré Y, Odulaja A, Beier JC, Githure JI, Yan G, Gouagna LC, Knols BG], Killeen GF: Behavioural determinants of gene flow in malaria vector populations: Anopheles gambiae males select large females as mates. Malar J 2002, I: I0.

68. Benedict MQ, Knols BG], Bossin HC, Howell PI, Mialhe E, Caceres C, Robinson AS: Colonization and mass rearing: learning from others. Malar J 2009, 8(Suppl 2):S4.

69. Chambers DL: Quality control in mass rearing. Annu Rev Entomol 1977, 22:289-308

70. Asman SM, Knop NF, Blomquist RE: Preliminary studies to identify selection factors in the laboratory colonization of Culex tarsalis. I Fla Anti-Mosq Assoc 1983, 54:16-2I.

7I. Haeger JS, O'Meara GF: Rapid incorporation of wild genotypes of Culex nigripalpus (Diptera: Culicidae) into laboratoryadapted strains. J Econ Entomol 1970, 63:1390-1391.

72. Knop NF, Asman SM, Reisen WK, Milby MM: Changes in the biology of Culex tarsalis (Diptera: Culicidae) associated with colonization under contrasting regimes. Environ Entomol 1987 16:405-4|4

73. McDonald PT: Effects of laboratory colonization on the reproductive abilities of a field-collected Culex tarsalis population. Edited by: Grant CD. USA: CMVCA Press; 1979:60-6I.

74. Kaneshiro KY: Sexual isolation, speciation and the direction of evolution. Evolution 1980, 34:437-444.

75. Bush GL, Neck RW, Kitto GB: Screwworm eradication: inadvertent selection for noncompetitive ecotypes during mass rearing. Science 1976, 193:491-493.

76. Miyatake T: Quantitative genetic aspects of the quality control of mass-reared insects: the case of the melon fly (Bactrocera cucurbitae). Formos Entomol 2006, 26:307-318.

77. Jones MDR, Cubbin CM, Marsh D: The circadian rhythm of flight activity of the mosquito Anopheles gambiae: the lightresponse rythm. J Exp Biol 1972, 57:337-346.

78. Baker RH, Reisen WK, Sakai RK, Rathor HR, Raana K, Azra K, Niaz S: Anopheles culicifacies: mating behavior and competitiveness in nature of males carrying a complex chromosomal aberration. Ann Entomol Soc Am 1980, 73:581-588.

79. Marchand RP: A new cage for observing mating behavior of wild Anopheles gambiae in the laboratory. I Am Mosq Control Assoc 1985, 1:234-236.

80. Boake CRB: Flying apart: mating behavior and speciation. Bio Science 2000, 50:50I-508.

8I. Rutledge LC, Ward RA, Bickley WE: Experimental hybridisation of geographic strains of Anopheles stephensi (Diptera: Culicidae). Ann Entomol Soc Am 1970, 63:1024-1030. 
82. Girod R, Coetzee M, Salvan M, Hunt RH: Polymorphisme chromosomique des populations d'Anopheles arabiensis (Diptera: Culicidae) de l'ile de la Reunion et inter-fertilite avec des populations d'Afrique continentale. Parasitol 200I, 43:99-103.

83. Cayol JP, Coronado P, Taher M: Sexual compatibility in medfly (Diptera: Tephritidae) from different origins. Fla Entomol 2002, 85:5।-57.

84. Reisen WK, Bock ME, Milby MM, Reeves WC: Attempted insertion of a recessive autosomal gene into a semi-isolated population of Culex tarsalis (Diptera: Culicidae). J Med Entomol 1985, 22:250-260.

85. Reisen WK, Milby MM, Asman SM, Bock ME, Meyer RP, McDonald PT, Reeves WC: Attempted suppression of a semi-isolated Culex tarsalis population by the release of irradiated males: a second experiment using males from a recently colonized strain. Mosq News 1982, 42:565-575.

86. Lima JBP, Valle D, Peixoto AA: Adaptation of a South American malaria vector to laboratory colonization suggests fastermale evolution for mating ability. BMC Evolu Biol 2004, 4:12.

87. Weidhaas DE, Schmidt CH, Seabrook EL: Field studies on the release of sterile males for the control of Anopheles quadrimaculatus. Mosq News 1962, 22:283-291.

88. Kaiser PE, Bailey DL, Lowe RE, Seawright JA, Dame DA: Mating competitiveness of chemosterilized males of a genetic sexing strain of Anopheles albimanus in laboratory and field tests. Mosq News 1979, 39:768-775.

89. Helinski ME, Hassan MM, El Motasim WM, Malcolm CA, Knols BG, El Sayed B: Towards a sterile insect technique field release of Anopheles arabiensis mosquitoes in Sudan: Irradiation, transportation, and field cage experimentation. Malar J 2008, 7:65.

90. Charlwood JD: May the force be with you: measuring mosquito fitness in the field. 2nd edition. Edited by: Takken W, Scott TW. Dordrecht: Kluwer Publisher; 2003:47-62.

91. Takken W, Constantini C, Dolo G, Hassanali A, Sagnon N, Osir E: Mosquito mating behavior. In Bridging Laboratory and Field Researcy for Genetic Control of Disease Vectors Edited by: Knols BG], Louis C. Netherlands: Springer; 2006:183-188.

92. Hendrichs J, Robinson AS, Cayol JP, Enkerlin W: Medfly areawide Sterile Insect Technique programmes for prevention, suppression or eradication: the importance of mating behavior studies. Fla Entomol 2002, 85: I-13.

93. Koyama J, Kakinohana H, Miyatake T: Eradication of the melon fly, Bactrocera cucurbitae, in Japan: importance of behaviour, ecology, genetics and evolution. Annu Rev Entomol 2004, 49:331-349.

94. Rull J, Brunel $O$, Mendez ME: Mass rearing history negatively affects mating success of male Anastrepha ludens (Diptera: tephritidae) reared for sterile insect technique programs. J Econ Entomol 2005, 98: $1510-1516$.

95. McCall PJ, Kelly DW: Learning and memory in disease vectors. Parasitology 2002, 18:429-433.

96. Robinson AS, Cayol JP, Hendrichs J: Recent findings on medfly sexual behavior: implications for SIT. Fla Entomol 2002, 85: $17|-| 8 \mid$.

97. Nunney L: Managing captive populations for release: a population genetic perspective. In Quality Control and production of Biological Control Agents: Theory and Testing Procedures Edited by: Van Lenteren JC. CAB International; 2003:73-88.

98. Panicker KN, Rajagolopan PK: Field observations on the swarming and the mating behaviour of Anopheles subpictus Grassi 1899. Indian J Med Res 1984, 80:60-62.
Publish with BioMed Central and every scientist can read your work free of charge

"BioMed Central will be the most significant development for disseminating the results of biomedical research in our lifetime. "

Sir Paul Nurse, Cancer Research UK

Your research papers will be:

- available free of charge to the entire biomedical community

- peer reviewed and published immediately upon acceptance

- cited in PubMed and archived on PubMed Central

- yours - you keep the copyright

Submit your manuscript here:

http://www.biomedcentral.com/info/publishing_adv.asp
BiolMedcentral 